\title{
Intelligent Business Transaction Agents for Cross-Organizational Workflow Definition and Execution
}

\author{
Mohammad Saleem ${ }^{1}$, Paul W.H. Chung ${ }^{1}$, Shaheen Fatima ${ }^{1}$, and Wei Dai ${ }^{2}$ \\ ${ }^{1}$ Computer Science Department, Loughborough University, Loughborough, LE11 3TU, UK \\ \{M.Saleem, P.W.H.Chung, S.S.Fatima\} alboro.ac.uk \\ ${ }^{2}$ School of Information Systems, Victoria University, Melbourne City MC, Victoria, Australia \\ Wei.Dai@vu.edu.au
}

\begin{abstract}
Business organizations seldom work in isolation; effective interaction and cooperation between different organizations is essential for the success of any organization. In order to work together, organizations must have compatible workflows. This paper proposes a framework for automatically creating compatible workflows from high level requirements given by organizations that intend to work together. The framework uses intelligent agents [1] for the creation and execution of workflows. The proposed framework is different from existing systems as they focus on collaboration by modifying existing workflows. The proposed framework will enable organizations to avoid the time consuming task of creating a workflow and then ensuring that it is compatible with every organization it interacts with.
\end{abstract}

Keywords. Cross-Organizational Workflow Collaboration, Web Service Composition, Workflow Generation.

\section{Introduction}

A business process represents successful work practice and is a crucial part of corporate asset. It refers to a set of connected and ordered activities to achieve a business goal within the context of an organizational structure [2]. In this internet age more and more organizations are embracing electronic commerce and there is increasing demand for automatic business process management. Also, when two organizations interact with each other, the need for automatic cross-organizational business process collaboration arises. Since workflow technology is commonly used for business process management within a single organization, there is a need for support for crossorganizational workflow collaboration.

For business collaboration to work, workflows of business partners should be compatible at the business level [3]. Compatible means that there should be an agreed sequence of exchanging collaborative messages and information. The points where collaborative messages and information is exchanged is called interface activity [4]. The set of all interface activities when extracted from a process is called an interface process. Considerable amount of effort is needed to ensure that workflows are compatible in the first place $[5,6]$. 
Fig. 1 shows a workflow for a vendor and the corresponding interface process, which only models the points where interaction takes place with another organization, the customer in this case. In this paper, an activity name followed by [s] or [r] means sending and receiving collaboration message respectively [4]. For example 'Advance Payment[r]' means an activity 'Advance Payment' is required to receive a message from the collaborating organization.

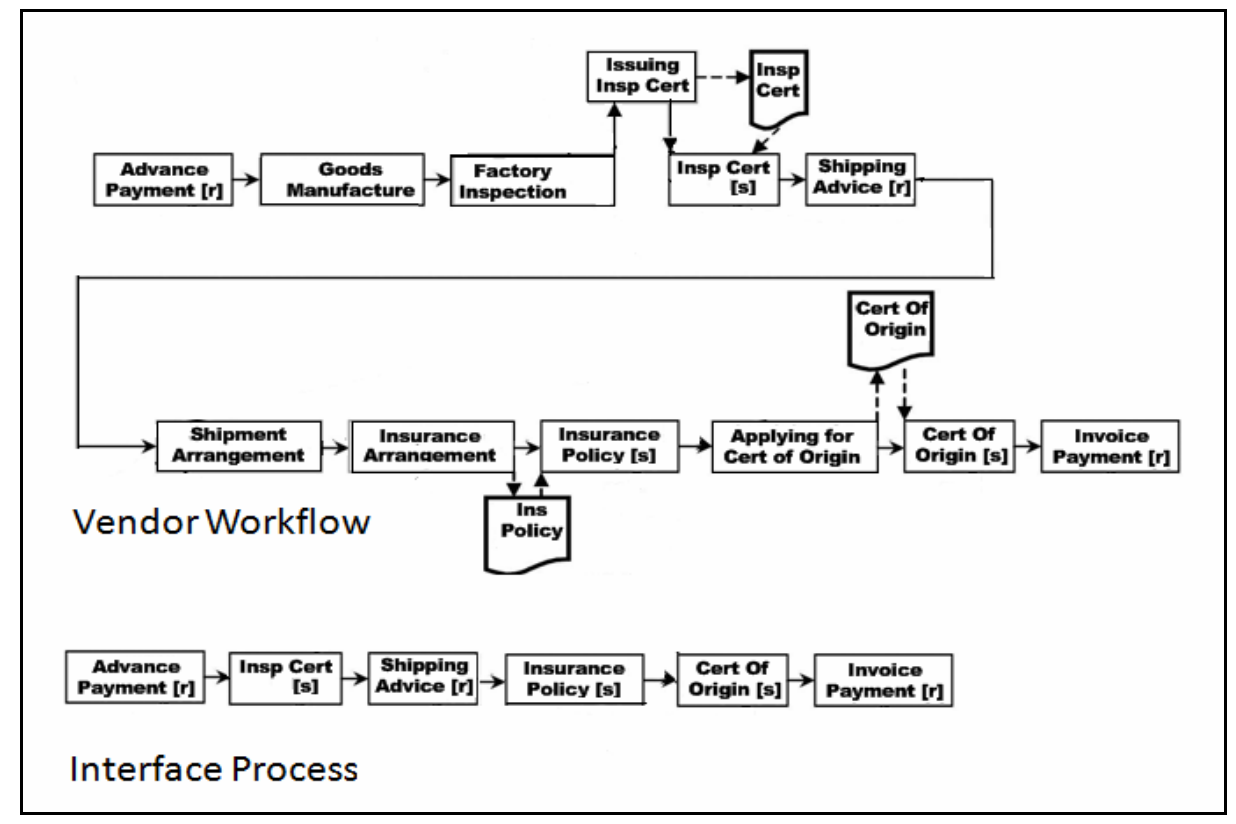

Fig. 1. Workflow and Interface Process for a Vendor

Any incompatibility between two workflows has to be reconciled before proceeding with the business. This can be a very time consuming process. To overcome this problem a new framework for automated workflow generation is presented.

Workflow generation is considered as an AI planning problem. AI planning creates an execution plan that reaches the required goal state from a given initial state. Since web services can be organized in a workflow to support the execution plan so AI planning can be applied to automatic web service composition and hence workflow generation [7]. Given a high level description of a goal, a planner can reason with all available services in terms of their capabilities. A required service that can achieve a desirable state will be identified and added into a workflow. Executing the complete workflow will result in the goal state [8].

Section 2 explains the proposed framework. Section 3 describes the implementation. Section 4 summarizes some related work. Conclusions are drawn in Section 5.

\section{Proposed Framework}

Fig. 2 shows the architecture of the proposed framework. Although the number of organizations is not limited to two, but for clarity the figure only depicts two 
organizations. Each organization has its own high level goals and OWLS process definition. Also for each organization a separate intelligent agent is created which has its own instance of SHOP2 planner.

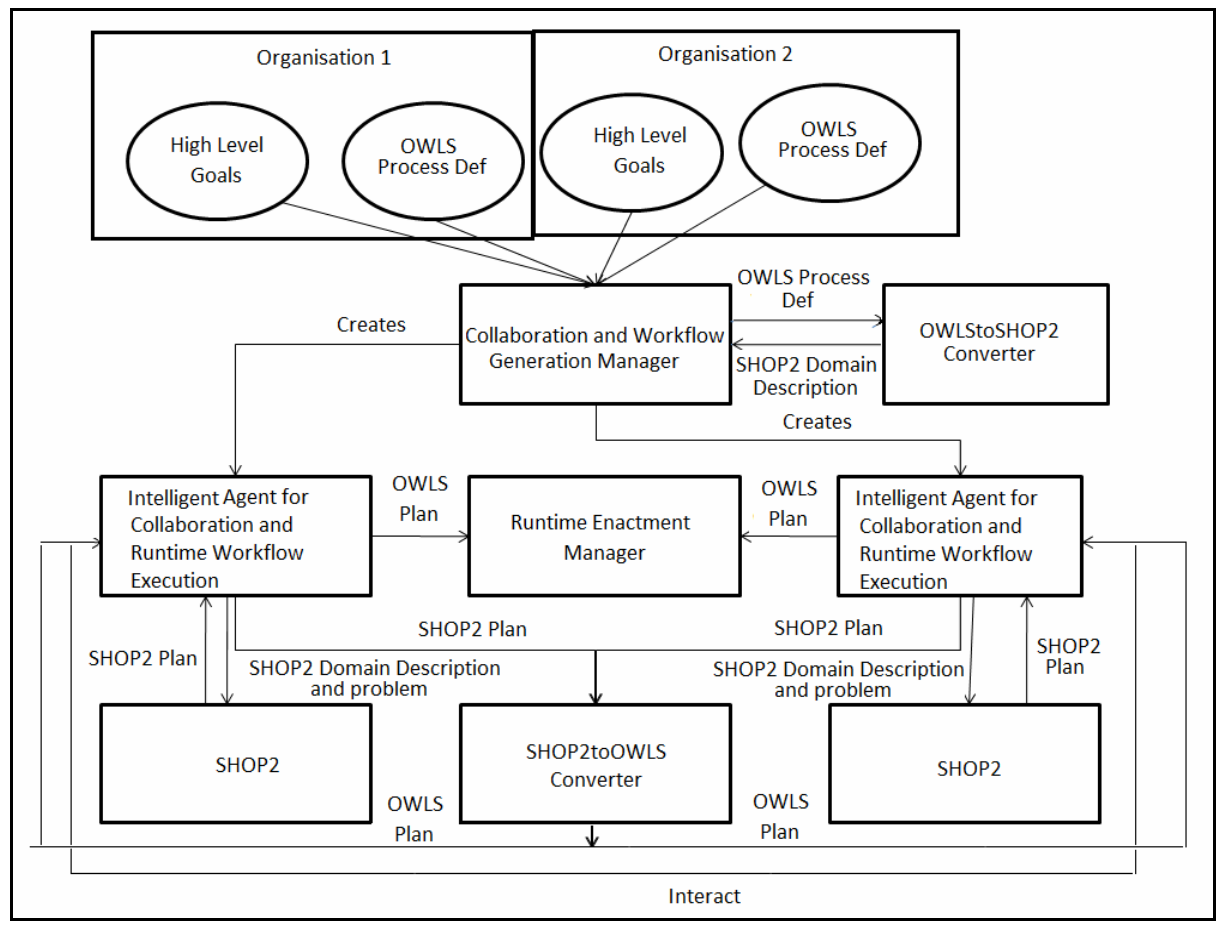

Fig. 2. Architectural Diagram

The following text discusses some of the basic components shown in Fig 2. Simple Hierarchical Ordered Planer 2 (SHOP2) $[9,10]$ is a domain independent hierarchical task network (HTN) planner which is used in the proposed system. It decomposes task into subtasks in the order they are to be executed. It requires domain description for planning. Domain description consists of operators and methods. Operators are primitive tasks while methods are specifications for decomposing composite tasks into subtasks [11].

As tasks are planned in the order in which they will be performed, this makes it possible for SHOP2 to know the current state of the world at every step. This in turn enables the precondition evaluation mechanism of SHOP2 to do reasoning and call external functions and sources [11]. A mechanism for querying external sources and backtracking in SHOP2 in case of failures is described in [12]. SHOP2 also allows tasks and subtasks to be ordered, unordered and partially ordered which makes it possible to interleave certain tasks with other tasks. These functionalities make SHOP2 an appropriate choice for cross-organizational workflow collaboration.

The OWL-Service (OWL-S) language provides a mechanism for describing web services [13]. It is used to describe the functionality, access point and execution 
mechanism of web services. OWL-S is a set of ontologies and OWL-S process ontology describes web services composition based on 'action' or 'process' metaphor. It describes simple tasks as simple actions or simple processes and complex tasks as composite actions or composite processes. This similar way of modeling makes it possible to translate OWL-S web services descriptions to SHOP2 domain descriptions.

Each organization has high level rules for carrying out business which are stated in its process definition. Process definition is actually the OWLS domain ontology of the respective organization. High level goals describe the final goals of the organization which have to be achieved. OWLS process definition and high level goals together express the business interest, requirements and rules of an organization to carry out business with another organization.

As shown in Fig. 2, the interacting organizations pass their OWL-S process definitions and high level goals to Collaboration and Workflow Generation Manager (CWGM). CWGM passes the process definitions to OWLS-SHOP2 translator which translates them into SHOP2 domain description. The translation algorithm was put forward and proved for soundness and correctness by Sirin et al. [11]. The simple processes are translated into operators while composite processes are translated into methods. OWLS-SHOP2 translator also translates high level goals into SHOP2 problem.

Intelligent agents are autonomous problem solving entities that can take input about the state of their environment and fulfill a specific role by acting on the environment in which they are situated [1]. Intelligent agents decentralize complex problems, define a simplified model to focus on specific details and define and manage the inter-relationships between different problem solving entities; hence making it easier to build complex software systems. CWGM creates an autonomous intelligent agent for each organization, which acts on behalf of its respective organization. Each agent has an instance of SHOP2 planner. The problem and the translated domain description are passed to the respective agents. Each agent will add a function call to itself in the precondition of each operator in the domain description of its respective organization. This way a step can only be added in a plan if the agent permits it to be added in the plan. The permission by the agent is based on its interaction with all other agents working on behalf of the interacting organizations. The agent only permits a step to be added in the plan if it does not make the plan incompatible with the plans of other interacting organizations. If a specific step makes the plan incompatible with the other plans then an alternative step is tried. The querying and backtracking mechanism presented by Au et al. is followed for this purpose [12].

The generated compatible SHOP2 plans are then transferred to SHOP2toOWLS converter to convert the plans from SHOP2 format into OWL-S format. OWL-S plans are passed back to the respective agents for execution with the help of Runtime Enactment Manager, which ensures that the transfer of information and files between the interacting organizations happens smoothly.

\section{Implementation}

The current prototype is able to create SHOP2 plans from high level user requirements and goals. The system takes a process definition and high level goals as input from user, translates the process definition into SHOP2 domain description and high 
level goals into SHOP2 problem. Then the system creates all possible plans from the SHOP2 domain description to solve the generated SHOP2 problem. The OWLSSHOP2 module from Transplan [14] is used in the system. Transplan is further based on the algorithm proposed by Sirin et al. [11]. The Java version of SHOP2 (JSHOP2) is used as a planner. Currently work is underway on extending the system to handle more than one organization so that based on high level requirements of interacting organizations, compatible workflows can be generated.

\section{Related Work}

Sirin et al.[15] presented a semi automatic system for web service composition. Sirin et al. [11] later extended his work to propose a fully automated system for web services composition. The system takes high level tasks, uses SHOP2 to create a plan to achieve them and then executes the plan using web services from the web. The authors have argued that web service composition is an AI planning problem. The framework proposed in this paper also generates all possible plans from an OWLS process definition and execute them using web services from the net. But unlike the proposed framework, system developed by Sirin et al. only targets web service composition for a single organization and does not take cross-organizational collaboration into account.

Chen and Chung [4] proposed a framework for cross-organizational workflow collaboration. The framework proposed by Chen and Chung detects incompatibilities between workflows, suggest changes to the workflows to remove the detected incompatibilities and ask the users in turn to see whether they would accept the suggested changes. Although their work saves considerable amount of time by automating workflow collaboration but users are still required to model workflows beforehand. Wang et al. [16] have also proposed a system for cross-organizational workflow collaboration and dynamic workflow composition. They use intelligent agents for discovering, executing and monitoring web services. They also use intelligent agents for dynamically composing workflows and negotiating over the net. Work done by Wang et al. is closely related to the work reported in this paper because it uses OWLS ontology to compose workflow at runtime and uses agents for negotiation. Wang et al. target collaboration among organization to take an alternative path in the workflow when certain service fails to achieve the desired goal in the workflow while the system proposed in this paper targets collaboration to generate compatible workflows from high level requirements.

\section{Conclusion}

This paper proposes a framework for cross-organizational workflow collaboration. It is different from existing systems because it automatically creates compatible workflows from high level goals and requirements from organizations that intend to collaborate. Other approaches aim to modify existing workflows of interacting organizations to make them compatible. This paper identifies workflow generation as AI planning problem and focuses on collaboration while generating the workflows. 


\section{References}

[1] Jennings, N.R.: An agent-based approach for building complex software systems. Communications of the ACM 44(4), 35-41 (2001)

[2] Workflow Management Coalition: Terminology \& Glossary. Technical Report WFMCTC-1011 (1999)

[3] Yang, J., Papazoglou, M.: Interoperation Support for Electronic Business. Communication of the ACM 43(6), 39-47 (2000)

[4] Chen, X., Chung, P.W.H.: Facilitating B2B E-Business by IT-Supported Business Process Negotiation Services. In: Proceedings of the 2008 IEEE International Conference on Service Operations and Logistics and Informatics, pp. 2800-2805 (2008)

[5] Schulz, K., Orlowska, M.: Facilitating cross-organizational workflows with a workflow view approach. Data and Knowledge Engineering 51(1), 109-147 (2004)

[6] Chiu, D.K.W., Cheung, S.C., Karlapalem, K., Li, Q., Till, S., Kafeza, E.: Workflow View Driven Cross-Organizational Interoperability in a Web-Services Environment. Information Technology and Management 5, 221-250 (2004)

[7] Dong, X., Wild, D.: An Automatic Drug Discovery Workflow Generation Tool using Semantic Web Technologies. In: Proceedings of Fourth IEEE International Conference on eScience, pp. 652-657 (2008)

[8] Chen, X., Yang, L.: Applying AI Planning to Semantic Web Services for Workflow Generation. In: Proceedings of the First International Conference on Semantics, Knowledge and Grid. IEEE Computer Society, Washington (2005)

[9] Nau, D., Muñoz-Avila, H., Cao, Y., Lotem, A., Mitchell, S.: Total-order planning with partially ordered subtasks. In: IJCAI 2001, Seattle (2001)

[10] Nau, D., Au, T., Ilghami, O., Kuter, U., Murdock, J., Wu, D., Yaman, F.: SHOP2: An HTN planning system. Journal of Artificial Intelligence Research, 379-404 (2003)

[11] Sirin, E., Parsia, B., Wu, D., Hendler, J., Nau, D.: HTN planning for web service composition using SHOP2. Journal of Web Semantics 1(4), 377-396 (2004)

[12] Au, T.C., Nau, D., Subrahmanian, V.S.: Utilizing Volatile External Information during Planning. In: Proceedings of the European Conference on Artificial Intelligence (ECAI), pp. 647-651 (2004)

[13] OWL Services Coalition: OWL-S: Semantic markup for web services (2003), http: / /www. daml.org/services/owl-s/1.0/

[14] Transplan, http://sourceforge.net/projects/transplan/

[15] Sirin, E., Hendler, J., Parsia, B.: Semi-automatic composition of Web services using semantic descriptions. In: Proceedings of Web Services: Modeling, Architecture and Infrastructure Workshop in Conjunction with ICEIS (2003)

[16] Wang, S.Y., Shen, W.M., Hao, Q.: An agent-based Web service workflow model for inter-enterprise collaboration. Expert System with Applications, 787-799 (2006) 